\title{
酞嗪并三唑或四唑类衍生物的合成及其抗惊厥活性研究
}

\author{
张海明张洪健田玉顺* 全哲山* \\ (延边大学药学院 延吉 133002)
}

\begin{abstract}
摘要 设计合成了 6-取代-1,2,4-三氮唑并[3,4- $a$ ] 酞嗪和 6-取代四氮唑并[5,1- $a$ ] 酞嗪四个系列 16 个衍生物. 其化学结构 均经过 ${ }^{1} \mathrm{H} N M R 、{ }^{13} \mathrm{C}$ NMR、IR 和 HRMS 确征. 药理方面, 利用最大电惊厥法(MES), 以小鼠腹腔给药测定其抗惊厥活 性. 实验结果显示 $N$-(4-溴苯基)-四唑 $\left[5,1-a\right.$ ] 酞嗪-6-胺(7a) 的抗惊厥活性最强, 其半数有效量 $\mathrm{ED}_{50}$ 为 $5.89 \mathrm{mg} \cdot \mathrm{kg}^{-1}$, 抗惊 厥作用强于对照药卡马西平.
\end{abstract}

关键词 唒嗪; 三唑; 四唑; 合成; 抗惊厥; 最大电惊厥法

\section{Synthesis and Anticonvulsant Activity Evaluation of Phthalazine and Heterocyclic Derivatives}

\author{
Zhang, Haiming Zhang, Hongjian Tian, Yushun* Quan, Zheshan* \\ (College of Pharmacy, Yanbian University, Yanji 133002)
}

\begin{abstract}
Four series of derivatives, 6-substituted-1,2,4-triazolo[3,4- $a$ ] phthalazine derivatives and 6-substituted tetrazolo[5,1-a]phthalazine derivatives have been synthesized. The structures of compounds are confirmed by ${ }^{1} \mathrm{H}$ NMR, ${ }^{13} \mathrm{C}$ NMR, IR and HRMS. The anticonvulsant effects of the compounds are evaluated with maximal electroshock test by intraperitoneally injected in mice. The experimental results show that $N$-(4-bromophenyl)tetrazolo[5,1-a]phthalazin-6-amine (7a) was the most potent compound, with a median effective dose of $5.89 \mathrm{mg} \cdot \mathrm{kg}^{-1}$. Its anticonvulsant effect is better than the reference drug, carbamazepine.
\end{abstract}

Keywords phthalazine; triazolo; tetrazolo; synthesis; anticonvulsant; the maximal electroshock

癫㾁是大脑神经元突发性异常放电，导致短暂大脑 功能障碍的一种慢性神经系统疾病，并且随患病时间的 推移反复发作; 癫㾁的发病原因非常复杂, 遗传、脑损 害与脑损伤、环境因素、发热、精神刺激等是常见的影 响因素 ${ }^{[1,2]}$. 据世界卫生组织(WHO)研究报告表明, 目前 全世界有超过 6000 万的患者饱受癫㾁疾病的困扰, 其 中 $80 \%$ 在发展中国家, 每年还出现 200 万新增癫痫病例 ${ }^{[3]}$. 手术治疗、针炎治疗和药物治疗等方法是常用的治 疗方法, 其中, 药物治疗是临床抗癫㾁的首选方法, 主 要防止和控制癫㾁的发作. 苯妥英、苯巴比妥、卡马西 平、丙戊酸等是目前临床常用的传统抗癫㾁药(AEDs). 20 世纪 80 年代以来, 国外开发上市的新抗瘨病药有加 巴喷丁、奥卡西平、托吡酯、拉莫三嗪等. 但应用现有 这些抗癫㾁药物治疗癫㾁，只能使 $60 \% \sim 70 \%$ 的癫㾁患 者的症状得到有效控制, 另有 $30 \% \sim 40 \%$ 的患者得不到
良好的效果 ${ }^{[4 \sim 7]}$. 而且如果长期服用这些药物, 会产生 全身性毒性作用、神经系统毒性、嗜睡、胃肠道紊乱和 巨幼红细胞性贫血等多样的副作用, 增加中毒、致畸的 风险 ${ }^{[8]}$. 因此, 研究低毒有效的新一代抗癫㾁药物仍具 有重要意义.

文献报道，酞嗪类化合物具有抗惊厥作用 ${ }^{[9]}$. 而前 期研究工作中, 我们发现三唑类和四唑类衍生物也具有 较好抗惊厥作用 ${ }^{[10 ~ 14]}$, 谭月德等 ${ }^{[15]}$ 在 2016 年发现 5-三 唑苯并 $[d]$ 檼唑类化合物具有较好的抗惊厥活性. 为了 获得抗惊厥作用更强的化合物, 本工作利用新药设计中 拼合原理，设计合成了 6-取代-1,2,4-三氮唑并[3,4- $a$ ] 酞 嗪和 6-取代四氮唑并 $[5,1-a]$ 酞嗪四个系列衍生物, 共 16 个新化合物. 其中, 酞嗪并三唑或四唑为结构主体, 而 6 位不同基团取代用于调节分子的疏水性，从而改变其 脂水分配系数, 以求达到更好的抗惊厥活性.

\footnotetext{
* Corresponding author. E-mail: ld-1@163.com

Received March 2, 2017; revised March 30, 2017; published online May 10, 2017.

Project supported by the National Natural Science Foundation of China (No. 21272005).

国家自然科学基金(No. 21272005)资助项目.
} 


\section{1 结果与讨论}

\section{1 目标化合物的合成}

目标物的合成参考文献 $[16 \sim 21]$ 方法，首先以双酮 酞嗪为起始原料, 用三氯氧磷进行氯代反应, 得到双氯 代产物 1. 化合物 1 再与水合肼发生肼代反应, 得到肼 代化合物 2. 化合物 2 与原甲酸三乙酯在 $100{ }^{\circ} \mathrm{C}$ 下发生 环合反应, 得到酞嗪并三唑中间体 3. 化合物 3 以 $N, N$ 二甲基甲酰胺为溶剂, 与各种吡啶胺类、苯胺类、苯硫 醇于 $100{ }^{\circ} \mathrm{C}$ 反应, 得到目标化合物 $\mathbf{5 a} \sim \mathbf{5 g}$ 和 $\mathbf{6 a} \sim \mathbf{6 c}$. 化合物 2 与亚硝酸钠, 以水作溶剂, 酸性条件发生重氮 化及环合反应, 生成酞嗪并四唑中间体 $\mathbf{4}$. 中间体 4 以 乙腈为溶剂, 于 $80{ }^{\circ} \mathrm{C}$ 下, 与各种苯胺类和苯硫醇类反 应得到酞嗪并四唑系列目标化合物 $7 \mathbf{a} \sim 7 \mathbf{c}$ 和 $8 \mathrm{a} \sim 8 \mathrm{c}$.<smiles>NNC1N=NC(Cl)c2ccccc21</smiles>

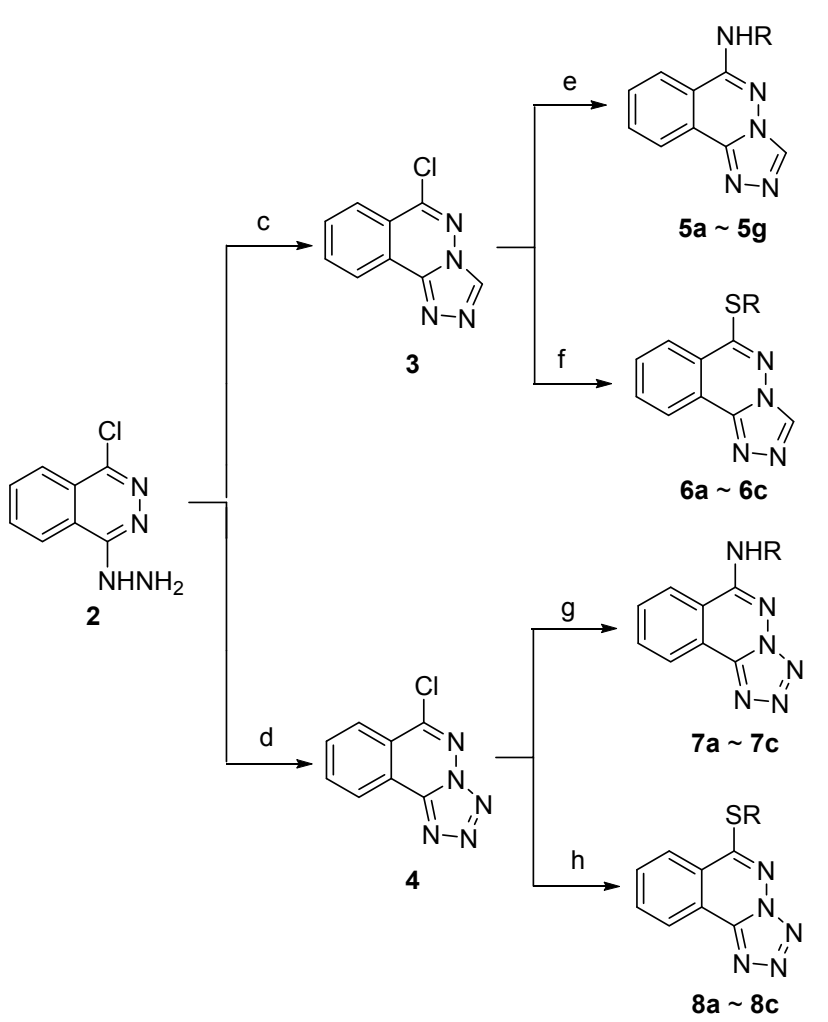

Reagents and conditions: (a) $\mathrm{POCl}_{3}, 80 \sim 110{ }^{\circ} \mathrm{C}, 75 \% \sim 80 \%$; (b) $\mathrm{NH}_{2} \mathrm{NH}_{2} \bullet \mathrm{H}_{2} \mathrm{O}, \mathrm{CH}_{3} \mathrm{CH}_{2} \mathrm{OH}$, reflux, $65 \% \sim 72 \%$; (c) $\mathrm{CH}\left(\mathrm{CH}_{3} \mathrm{CH}_{2} \mathrm{O}\right)_{3}$, $100{ }^{\circ} \mathrm{C}, 70 \% \sim 80 \%$; (d) $\mathrm{H}_{2} \mathrm{O}, \mathrm{HCl}, \mathrm{NaNO}_{2},<5{ }^{\circ} \mathrm{C}, 65 \% \sim 72 \%$; (e) $\mathrm{RNH}_{2}, \mathrm{DMF}, \mathrm{NaOH}, 100{ }^{\circ} \mathrm{C}$; (f) $\mathrm{RSH}, \mathrm{DMF}, \mathrm{NaOH}, 100{ }^{\circ} \mathrm{C}$; (g) $\mathrm{RNH}_{2}, \mathrm{CH}_{3} \mathrm{CN}, \mathrm{K}_{2} \mathrm{CO}_{3}$, reflux; (h) $\mathrm{RSH}, \mathrm{CH}_{3} \mathrm{CN}, \mathrm{K}_{2} \mathrm{CO}_{3}$, reflux

图式 1 酞嗪衍生物的合成路线

Scheme 1 Synthesis of phthalazine derivatives

\section{2 化合物的生物活性分析}

通过 I 期药理实验，初步评价了目标化合物 $5 \mathbf{a} \sim 5 \mathrm{~g}$, $6 a \sim 6 c, 7 a \sim 7 c$ 和 8a $\sim 8 c$ 的抗惊厥活性. 如表 1 所示, 在 $300 \mathrm{mg} \cdot \mathrm{kg}^{-1}$ 剂量下, 大多数化合物都显示了不同程

表 1 化合物的小鼠抗惊厥作用 $\mathrm{I}$ 期实验数据 ${ }^{a}$

Table 1 Phase I anticonvulsant data of compounds in mice

\begin{tabular}{ll}
\hline Compd. & $\mathrm{R}$ \\
\cline { 2 - 2 } & $30 \mathrm{mg} \cdot \mathrm{kg}^{-1} 100 \mathrm{mg} \cdot \mathrm{kg}^{-1} 300 \mathrm{mg} \cdot \mathrm{kg}^{-1}$ \\
\hline
\end{tabular}

$5 a$

$5 \mathbf{b}$<smiles>Cc1ccc(F)cn1</smiles>

- $\quad 0 / 3$

$0 / 3$

$1 / 3$

$1 / 3$

$2 / 3$

$5 \mathbf{c}$<smiles>Cc1cccc(F)c1</smiles>

5d<smiles>Cc1cccc(Br)c1</smiles>

$0 / 3$

$1 / 3$

$0 / 3$

$2 / 3$

$5 e$<smiles>Cc1ccc(C(F)(F)F)cc1</smiles>

-<smiles>Cc1ccc(Cl)c(Cl)c1</smiles>

-

$0 / 3$

$5 g$

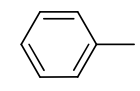

$2 / 3$

$3 / 3$

$3 / 3$

6a<smiles>Cc1ccc(Br)cc1</smiles>

$0 / 3$

$2 / 3$<smiles>Cc1ccc(Cl)cc1</smiles>

$3 / 3$

$3 / 3$<smiles>Cc1ccc(F)cc1</smiles>

$1 / 3$

$3 / 3$<smiles>Cc1ccc(Br)cc1</smiles>

$3 / 3$

$3 / 3$

$0 / 3$

$2 / 3$

$7 c$<smiles>Cc1ccc(F)cc1</smiles>

$1 / 3$

$2 / 3$

$8 \mathbf{a}$<smiles>Cc1ccc(Br)cc1</smiles><smiles>C1CCCCC1</smiles>

$0 / 3$

$\mathbf{8 b}$<smiles>Cc1ccc(Cl)cc1</smiles><smiles>C=CC</smiles>

$0 / 3$

8c<smiles>Cc1ccc(F)cc1</smiles>

$0 / 3$

$1 / 3$ tested; "—" : not test. 
度的抗惊厥活性, 而在 $100 \mathrm{mg} \cdot \mathrm{kg}^{-1}$ 剂量下, 只有部分 化合物表现出明显的抗惊厥活性，其中化合物 $\mathbf{5 c}, \mathbf{5 g}$, $\mathbf{6 a} \sim 6 \mathbf{c}, 7 \mathbf{a} \sim 7 \mathrm{c}$ 表现最为突出, 化合物 $6 \mathbf{b}$ 和 $7 \mathbf{a}$ 在 30 $\mathrm{mg} \cdot \mathrm{kg}^{-1}$ 仍表现出显著的抗惊厥活性. 在酞嗪并三唑系 列化合物中，与硫醇反应后的化合物 $\mathbf{6}$ 其抗惊厥活性明 显优于与芳胺反应后的产物 $\mathbf{5}$; 酞嗪并四唑系列化合物 $7 a \sim 7 c$ 和 8a $\sim 8 c$ 药理结果显示, 其结构中的 $\mathrm{NH}$ 被 $\mathrm{S}$ 替代后使化合物的抗惊厥活性减弱, 说明 $\mathrm{NH}$ 结构对化 合物的抗惊厥活性影响较大. 化合物 $\mathbf{8}$ 与化合物 $\mathbf{6}$ 相比, 四唑环替代三唑环后, 其活性也呈现减弱趋势, 说明四 唑环对化合物的抗惊厥效能不如三唑环高. 对化合物 7a 活性最优的探讨主要分为两个方面: 一方面, 化合物 6a, 7a 和 8a 的取代 $\mathrm{R}$ 基团上都是 $p$ - $\mathrm{Br}$, 化合物 6a 与 $8 \mathrm{a}$ 相比, 化合物 6a 的活性优于化合物 8a, 说明化合物中 三唑基团对抗惊厥活性的效能优于四唑基团, 而化合物 $7 \mathbf{a}$ 与 $8 \mathbf{a}$ 的区别在于 $\mathrm{NH}$ 取代 $\mathrm{S}$, 说明 $\mathrm{NH}$ 的抗惊厥效能 更高, 然而, 6a 抗惊厥活性却不如 7a, 说明可能 NH 对 抗惊厥的活性效能优于三唑环的效能, NH 是关键活性 基团, 可能与某些酶发生相互作用而发挥抗惊厥活性; 另一方面, 化合物 7a $\sim \mathbf{7}$ 对位分别是 $\mathrm{Br}, \mathrm{Cl}, \mathrm{F}$ 原子, 而 化合物 7a 取代基对位恰巧为吸电子能力处于中间的 $\mathrm{Br}$ 原子, 则说明化合物 $7 \mathbf{a}$ 的活性最好可能是因为各基团 效能综合的结果使此时的脂水分配系数最适合透过血 脑屏障从而发挥抗惊厥作用.

根据 I 期药理实验的结果, 进一步进行了 II 期药理 实验, 对具有较好活性的化合物 $6 \mathrm{~b}$ 和 7a 进行研究, 以 定量评价其抗惊厥活性. 2 个目标化合物和阳性对照药 卡马西平的实验结果见表 2, 化合物 $\mathbf{6 b}$ 和 $7 \mathbf{a}$ 的 $\mathrm{ED}_{50}$ 分 别为 14.94 和 $5.84 \mathrm{mg} \cdot \mathrm{kg}^{-1}$, 其中化合物 $\mathbf{6 b}$ 活性稍弱于 阳性对照药卡马西平, 而化合物 $7 \mathbf{a}$ 的 $\mathrm{ED}_{50}$ 明显低于阳 性对照药卡马西平 $(11.80 \mathrm{mg} / \mathrm{kg})$, 具有进一步开发的价 值.

表 2 化合物 $5 \mathbf{i}$ 和 $6 \mathbf{d}$ 的小鼠抗惊厥作用 II 期实验数据

Table 2 Phase II anticonvulsant data of compounds $5 \mathbf{i}$ and $\mathbf{6 d}$ in mice

\begin{tabular}{ccc}
\hline Compd. & $\mathrm{R}$ & $\mathrm{ED}_{50} /\left(\mathrm{mg} \bullet \mathrm{kg}^{-1}\right)$ \\
\hline $\mathbf{6 b}$ & $14.94(11.78 \sim 20.92)^{a}$ \\
$7 \mathbf{a}$ & \\
Carbamazepine & $11.80(8.96 \sim 12.81)^{a}$ \\
\hline${ }^{a}$ 95\% confidence intervals given in parentheses.
\end{tabular}

\section{2 结论}

以双酮唒嗪为先导化合物, 经氯代、肼代、环合, 获
得双酮呔嗪并三唑或四唑中间体，然后在其 6 位引入不 同取代基，最终得到四个系列 16 个目标化合物，所合成 化合物结构均经过 ${ }^{1} \mathrm{H} N M R 、{ }^{13} \mathrm{C}$ NMR、IR 和 HRMS 确证. 在对抗最大电惊厥(MES)法实验中, 化合物 7a 的 抗惊厥活性最为显著, 其 $\mathrm{ED}_{50}$ 为 $5.89 \mathrm{mg} / \mathrm{kg}$, 明显优于 阳性对照药卡马西平, 对研究开发新型抗癫㾁候选药物 具有重要意义.

\section{3 实验部分}

\section{1 仪器与试剂}

熔点用毛细管法测定, 温度计未经校正; ${ }^{1} \mathrm{H}$ NMR 和 ${ }^{13} \mathrm{C}$ NMR 用 BRUKER AV-300 型核磁共振仪器测定 (TMS 为内标, $\mathrm{CDCl}_{3}$ 或 DMSO- $d_{6}$ 为溶剂); IR 谱采用 FI-1730 型红外光谱仪 ( $\mathrm{KBr}$ 压片)测定; HRMS 数据来源 于 MALDI-TOF/TOF; 本实验所用试剂均为分析纯或化 学纯.

\section{2 化合物的合成}

\subsubsection{1,4-二氯酞嗪(1)和 1-肼基-4-氯酞嗪(2)的合成}

按照文献[22]方法制备, 合成方法与数据表征与文 献一致.

3.2.2 6-氯-[1,2,4]三氮唑并 $[3,4-a]$ 酞嗪 (3)的合成

将化合物 $2(2 \mathrm{~g}, 10 \mathrm{mmol})$ 溶解在 $60 \mathrm{~mL}$ 的原甲酸三 乙酯中, $150{ }^{\circ} \mathrm{C}$ 搅拌回流 $2 \mathrm{~h}$, 薄层色谱(TLC) $\left(V_{\mathrm{DCM}}\right.$ : $\left.V_{\mathrm{MeOH}}=30: 1\right)$ 显示反应完反应, 减压蒸出溶剂, 水洗干 燥, 硅胶柱层析 (二氯甲烷)得化合物 $\mathbf{3}$ 为白色固体, 产 率: $70 \% \sim 80 \%$. m.p. $177 \sim 179{ }^{\circ} \mathrm{C}$ (文献值 ${ }^{[22]}: 176 \sim$ $\left.177{ }^{\circ} \mathrm{C}\right) ;{ }^{1} \mathrm{H}$ NMR (300 MHz, $\left.\mathrm{CDCl}_{3}\right) \delta$ : $9.01(\mathrm{~s}, 1 \mathrm{H}), 8.73$ (d, $J=7.9 \mathrm{~Hz}, 1 \mathrm{H}), 8.30(\mathrm{~d}, J=8.2 \mathrm{~Hz}, 1 \mathrm{H}), 8.05$ (td, $J=$ $7.8,1.2 \mathrm{~Hz}, 1 \mathrm{H}), 7.96 \sim 7.88(\mathrm{~m}, 1 \mathrm{H})$.

\subsection{3 化合物 $5 \mathrm{a} \sim 5 \mathrm{~g}$ 和 $6 \mathrm{a} \sim 6 \mathrm{c}$ 的合成通法}

以 $N$-(5-氟吡啶-2-基)-1,2,4-三氮唑并 [3,4- $a$ ]酞嗪-6胺 $(5 \mathrm{a})$ 的合成为例. 将化合物 $4(0.3 \mathrm{~g}, 1.5 \mathrm{mmol})$ 、氢氧 化钠 $(0.07 \mathrm{~g}, 1.8 \mathrm{mmol})$ 和 2 -氨基-5-氟吡啶 $(0.2 \mathrm{~g}, 1.8$ $\mathrm{mmol}$ )分散在 $5 \mathrm{~mL}$ 的 $\mathrm{N}, \mathrm{N}$-二甲基甲酰胺(DMF)中, $100{ }^{\circ} \mathrm{C}$ 下搅拌反应 $2 \mathrm{~h}, \mathrm{TLC}\left(V_{\mathrm{DCM}}: V_{\mathrm{MeOH}}=30: 1\right)$ 显示 反应结束, 减压蒸出大部分 $\mathrm{DMF}$, 加入 $50 \mathrm{~mL}$ 冰水, 析 出沉淀, 减压抽滤得滤饼, 水洗 3 遍, 干燥, 用乙醇重结 晶得纯品 $\mathbf{5 a}$.

$N$-(5-氟吡啶-2-基)-1,2,4-三氮唑并 [3,4- $a$ ] 酞嗪-6-胺 (5a): 棕色固体, 产率 40.9\%. m.p. 300 304 ${ }^{\circ} \mathrm{C} ;{ }^{1} \mathrm{H}$ NMR (300 MHz, DMSO- $d_{6}$ ) $\delta$ : 10.03 (s, 1H, NH), 9.28 (s, $1 \mathrm{H}, \mathrm{CH}), 8.64$ (d, $J=7.7 \mathrm{~Hz}, 1 \mathrm{H}, \operatorname{ArH}), 8.50$ (d, $J=7.4$ $\mathrm{Hz}, 1 \mathrm{H}, \operatorname{ArH}), 8.40$ (s, 1H, PyH), 8.14 7.98 (m, 2H, Py-H), 7.91 (d, $J=7.6 \mathrm{~Hz}, 1 \mathrm{H}, \mathrm{ArH}), 7.81$ (s, 1H, ArH); 
${ }^{13} \mathrm{C}$ NMR (75 MHz, DMSO- $\left.d_{6}\right) \delta$ : 154.24, 149.72, 141.67, 139.88, 135.68, 135.35, 134.01, 131.04, 125.91, 125.73, 125.48, 123.90, 123.17, 119.43, 116.51, 110.36; IR (KBr) $v: 3262.86(\mathrm{~N}-\mathrm{H}), 1520(\mathrm{C}=\mathrm{N}) \mathrm{cm}^{-1}$; ESI-HRMS calcd for $\mathrm{C}_{14} \mathrm{H}_{10} \mathrm{FN}_{6}[\mathrm{M}+\mathrm{H}]^{+}$281.0951, found 281.0935.

$N$-(6-氯吡啶-3-基)-1,2,4-三氮唑并 $[3,4-a$ ] 酞嗪-6-胺 (5b): 合成方法及投料比同上, 产品为棕色固体, 产率 40.9\%. m.p. $320 \sim 322{ }^{\circ} \mathrm{C}$; ${ }^{1} \mathrm{H}$ NMR (300 MHz, DMSO$\left.d_{6}\right) \delta: 9.58(\mathrm{~s}, 1 \mathrm{H}, \mathrm{NH}), 9.27(\mathrm{~s}, 1 \mathrm{H}, \mathrm{CH}), 8.86(\mathrm{~d}, J=2.5$ $\mathrm{Hz}, 1 \mathrm{H}, \mathrm{Py}-\mathrm{H}$ ), 8.57 (d, $J=8.0 \mathrm{~Hz}, 1 \mathrm{H}, \mathrm{ArH}), 8.49$ (d, $J=$ $7.7 \mathrm{~Hz}, 1 \mathrm{H}, \mathrm{ArH}), 8.28$ (dd, $J=8.7,2.7 \mathrm{~Hz}, 1 \mathrm{H}, \mathrm{Py}-\mathrm{H})$, $8.03(\mathrm{t}, J=7.5 \mathrm{~Hz}, 1 \mathrm{H}, \mathrm{ArH}), 7.95(\mathrm{t}, J=7.7 \mathrm{~Hz}, 1 \mathrm{H}, \mathrm{ArH})$, $7.54(\mathrm{~d}, J=8.7 \mathrm{~Hz}, 1 \mathrm{H}$, Py-H$) ;{ }^{13} \mathrm{C}$ NMR $(75 \mathrm{MHz}$, DMSO- $\left.d_{6}\right) \delta: 149.89,143.77,142.92,141.53,139.97$, $136.62,134.10,132.31,131.08,125.23,124.36,123.83$, 123.36, 119.15; IR (KBr) v: $3282.28(\mathrm{~N}-\mathrm{H}), 1517.14$ $(\mathrm{C}=\mathrm{N}) \mathrm{cm}^{-1}$; ESI-HRMS calcd for $\mathrm{C}_{14} \mathrm{H}_{10} \mathrm{ClN}_{6}[\mathrm{M}+\mathrm{H}]^{+}$ 297.0655, found 297.0641.

$N$-(3-氟苯基)-1,2,4-三氮唑并 $[3,4-a]$ 酞嗪-6-胺 $(\mathbf{5 c})$ : 合成方法及投料比同上, 产品为淡黄色粉末, 产率 65.9\%. m.p. $314 \sim 318{ }^{\circ} \mathrm{C} ;{ }^{1} \mathrm{H}$ NMR $(300 \mathrm{MHz}$, DMSO$\left.d_{6}\right) \delta: 9.42(\mathrm{~s}, 1 \mathrm{H}, \mathrm{NH}), 9.30(\mathrm{~s}, 1 \mathrm{H}, \mathrm{CH}), 8.59(\mathrm{~d}, J=7.6$ $\mathrm{Hz}, 1 \mathrm{H}, \mathrm{ArH}), 8.48(\mathrm{~d}, J=7.3 \mathrm{~Hz}, 1 \mathrm{H}, \mathrm{ArH}), 8.01$ (t, $J=$ $7.1 \mathrm{~Hz}, 1 \mathrm{H}, \mathrm{ArH}), 7.95$ (d, $J=7.4 \mathrm{~Hz}, 1 \mathrm{H}, \mathrm{ArH}), 7.79$ (d, $J=11.7 \mathrm{~Hz}, 1 \mathrm{H}, \mathrm{ArH}), 7.61$ (d, $J=7.8 \mathrm{~Hz}, 1 \mathrm{H}, \mathrm{ArH}), 7.41$ (dd, $J=14.7,7.4 \mathrm{~Hz}, 1 \mathrm{H}, \mathrm{ArH}), 6.91$ (t, $J=6.9 \mathrm{~Hz}, 1 \mathrm{H}$, ArH); ${ }^{13} \mathrm{C}$ NMR (75 MHz, DMSO- $d_{6}$ ) $\delta: 164.24,149.82$, $142.02,141.47,140.02,133.93,131.00,130.55,125.26$, $123.83,123.31,119.31,117.26,109.91,109.62,108.42$, 108.07; IR (KBr) v: $3237.14(\mathrm{~N}-\mathrm{H}), 1514.29(\mathrm{C}=\mathrm{N})$ $\mathrm{cm}^{-1}$; ESI-HRMS calcd for $\mathrm{C}_{15} \mathrm{H}_{11} \mathrm{FN}_{5}[\mathrm{M}+\mathrm{H}]^{+}$ 280.0988, found 280.0991 .

$N$-(3-溴苯基)-1,2,4-三氮唑并 [3,4- $a$ ]酞嗪-6-胺 $(\mathbf{5 d})$ : 合成方法及投料比同上, 产品为棕色固体, 产率 54.0\%. m.p. 302 304 ${ }^{\circ} \mathrm{C} ;{ }^{1} \mathrm{H}$ NMR (300 MHz, DMSO$\left.d_{6}\right) \delta: 9.39(\mathrm{~s}, 1 \mathrm{H}, \mathrm{NH}), 9.27(\mathrm{~s}, 1 \mathrm{H}, \mathrm{CH}), 8.59(\mathrm{~d}, J=7.6$ $\mathrm{Hz}, 1 \mathrm{H}, \mathrm{ArH}), 8.50(\mathrm{~d}, J=7.2 \mathrm{~Hz}, 1 \mathrm{H}, \mathrm{ArH}), 8.08 \sim 7.91$ (m, 3H, ArH), 7.86 (d, J=7.3 Hz, 1H, ArH), $7.45 \sim 7.21$ $(\mathrm{m}, 2 \mathrm{H}, \mathrm{ArH}) ;{ }^{13} \mathrm{C}$ NMR $\left(75 \mathrm{MHz}, \mathrm{DMSO}-d_{6}\right) \delta: 149.90$, $141.89,141.52$, 139.98, 133.97, 131.04, 126.02, 125.27, 123.94, 123.85, 123.33, 121.87, 120.49, 120.0, 119.34; IR (KBr) v: $3188.57(\mathrm{~N}-\mathrm{H}), 1514.29(\mathrm{C}=\mathrm{N}) \mathrm{cm}^{-1}$; ESIHRMS calcd for $\mathrm{C}_{15} \mathrm{H}_{11} \mathrm{BrN}_{5}[\mathrm{M}+\mathrm{H}]^{+} 340.0198$, found 340.0190 .

$N$-(4-三氟甲基苯基)-1,2,4-三氮唑并 [3,4- $a$ ]酞嗪-6-
胺 (5e): 合成方法及投料比同上，产品为白色粉末，产 率 12.3\%. m.p. $296 \sim 298{ }^{\circ} \mathrm{C} ;{ }^{1} \mathrm{H}$ NMR $(300 \mathrm{MHz}$, DMSO- $\left.d_{6}\right) \delta: 9.65(\mathrm{~s}, 1 \mathrm{H}, \mathrm{NH}), 9.29(\mathrm{~s}, 1 \mathrm{H}, \mathrm{CH}), 8.62(\mathrm{~d}$, $J=8.0 \mathrm{~Hz}, 1 \mathrm{H}, \mathrm{ArH}), 8.56 \sim 8.46(\mathrm{~m}, 1 \mathrm{H}, \mathrm{ArH}), 8.09 \sim$ $7.93(\mathrm{~m}, 4 \mathrm{H}, \mathrm{ArH}), 7.74(\mathrm{~d}, J=8.8 \mathrm{~Hz}, 2 \mathrm{H}, \operatorname{ArH}) ;{ }^{13} \mathrm{C}$ NMR (75 MHz, DMSO- $d_{6}$ ) $\delta$ : 149.80, 144.07, 141.57, $139.97,134.08,131.05,126.32,126.27,125.43,123.89$, 123.33, 121.16, 119.39; IR (KBr) v: $3288.57(\mathrm{~N}-\mathrm{H})$, $1517.72(\mathrm{C}=\mathrm{N}) \mathrm{cm}^{-1}$; ESI-HRMS calcd for $\mathrm{C}_{16} \mathrm{H}_{11} \mathrm{~F}_{3} \mathrm{~N}_{5}$ $[\mathrm{M}+\mathrm{H}]^{+}$330.0967, found 330.0953.

$N$-(3,4-二氯苯基)-1,2,4-三氮唑并 [3,4- $a$ ] 酞嗪-6-胺 (5f): 合成方法及投料比同上，产品为黄色粉末，产率 77.1\%. m.p. $325 \sim 330{ }^{\circ} \mathrm{C} ;{ }^{1} \mathrm{H}$ NMR (300 MHz, DMSO$\left.d_{6}\right) \delta: 9.52(\mathrm{~s}, 1 \mathrm{H}, \mathrm{NH}), 9.30(\mathrm{~s}, 1 \mathrm{H}, \mathrm{CH}), 8.58(\mathrm{~d}, J=8.1$ $\mathrm{Hz}, 1 \mathrm{H}, \mathrm{ArH}), 8.54 \sim 8.47$ (m, $1 \mathrm{H}, \mathrm{ArH}), 8.13$ (d, $J=2.5$ $\mathrm{Hz}, 1 \mathrm{H}, \mathrm{ArH}), 8.04$ (t, $J=7.5 \mathrm{~Hz}, 1 \mathrm{H}, \mathrm{ArH}), 7.96$ (t, $J=7.0$ $\mathrm{Hz}, 1 \mathrm{H}, \mathrm{ArH}), 7.83$ (dd, $J=8.9,2.5 \mathrm{~Hz}, 1 \mathrm{H}, \mathrm{ArH}), 7.63(\mathrm{~d}$, $J=8.8 \mathrm{~Hz}, 1 \mathrm{H}, \mathrm{ArH}) ;{ }^{13} \mathrm{C}$ NMR $\left(75 \mathrm{MHz}, \mathrm{DMSO}-d_{6}\right) \delta$ : $149.77,141.51,140.46,140.01,135.60,134.06,131.29$, $131.06,130.85,125.31,124.74,123.85,123.34,122.71$, 121.58, 119.28; IR (KBr) v: $3237.14(\mathrm{~N}-\mathrm{H}), 1514.29(\mathrm{C}$ $=\mathrm{N}) \mathrm{cm}^{-1}$; ESI-HRMS calcd for $\mathrm{C}_{15} \mathrm{H}_{10} \mathrm{Cl}_{2} \mathrm{~N}_{5}[\mathrm{M}+\mathrm{H}]^{+}$ 330.0313 , found 330.0301 .

$N$-苯基-1,2,4-三氮唑并 [3,4- $a$ ]酞嗪-6-胺(5g): 合成 方法及投料比同上，产品为淡黄色粉末，产率 $20.6 \%$. m.p. $260 \sim 263{ }^{\circ} \mathrm{C} ;{ }^{1} \mathrm{H}$ NMR (300 MHz, DMSO- $\left.d_{6}\right) \delta$ : $9.28(\mathrm{~s}, 1 \mathrm{H}, \mathrm{NH}), 9.22(\mathrm{~s}, 1 \mathrm{H}, \mathrm{CH}), 8.60(\mathrm{~d}, J=8.0 \mathrm{~Hz}, 1 \mathrm{H}$, ArH), 8.48 (d, $J=7.7 \mathrm{~Hz}, 1 \mathrm{H}, \mathrm{ArH}), 8.00$ (t, $J=7.4 \mathrm{~Hz}$, 1H, ArH), 7.92 (t, $J=7.7 \mathrm{~Hz}, 1 \mathrm{H}, \operatorname{ArH}), 7.79$ (d, $J=7.6$ $\mathrm{Hz}, 2 \mathrm{H}, \mathrm{ArH}), 7.39$ (t, $J=7.9 \mathrm{~Hz}, 2 \mathrm{H}, \mathrm{ArH}), 7.11$ (t, $J=7.3$ $\mathrm{Hz}, 1 \mathrm{H}, \mathrm{ArH}) ;{ }^{13} \mathrm{C}$ NMR $\left(75 \mathrm{MHz}, \mathrm{DMSO}-d_{6}\right) \delta: 150.22$, $141.52,140.10,139.89,133.76,130.97,129.09,125.26$, $123.80,123.69,123.27,122.16,119.43$; IR $(\mathrm{KBr}) v$ : $3351.7(\mathrm{~N}-\mathrm{H}), 1517.14(\mathrm{C}=\mathrm{N}) \mathrm{cm}^{-1}$; ESI-HRMS calcd for $\mathrm{C}_{15} \mathrm{H}_{12} \mathrm{~N}_{5}[\mathrm{M}+\mathrm{H}]^{+}$262.1093, found 262.1079

6-(4-溴代硫苯基)-1,2,4-三氮唑并 [3,4- $a$ ] 酞嗪(6a): 合成方法及投料比同上, 产品为淡黄色固体, 产率 23.1\%. m.p. $170 \sim 174{ }^{\circ} \mathrm{C} ;{ }^{1} \mathrm{H}$ NMR (300 MHz, $\left.\mathrm{CDCl}_{3}\right) \delta$ : 8.77 (s, 1H, CH), 8.70 (d, J=7.9 Hz, 1H, ArH), 8.23 (d, $J=8.2 \mathrm{~Hz}, 1 \mathrm{H}, \mathrm{ArH}), 8.08 \sim 7.94(\mathrm{~m}, 1 \mathrm{H}, \mathrm{ArH}), 7.90 \sim$ $7.81(\mathrm{~m}, 1 \mathrm{H}, \mathrm{ArH}), 7.73 \sim 7.61(\mathrm{~m}, 2 \mathrm{H}, \mathrm{ArH}), 7.58 \sim 7.50$ $(\mathrm{m}, 2 \mathrm{H}, \mathrm{ArH}) ;{ }^{13} \mathrm{C}$ NMR $\left(75 \mathrm{MHz}, \mathrm{CDCl}_{3}\right) \delta: 157.33$, $137.15,134.10,132.85,130.94,125.62,125.47,124.87$, 123.79, 122.86, 122.63; IR (KBr) v: $1502.86(\mathrm{C}=\mathrm{N}) \mathrm{cm}^{-1}$; ESI-HRMS calcd for $\mathrm{C}_{15} \mathrm{H}_{10} \mathrm{BrN}_{4} \mathrm{~S}[\mathrm{M}+\mathrm{H}]^{+} 356.9810$, 
found 356.9814 .

6-(4-氯代硫苯基)-1,2,4-三氮唑并 $[3,4-a]$ 酞嗪(6b): 合成方法及投料比同上, 产品为黄色固体, 产率 $23.9 \%$. m.p. $166 \sim 168{ }^{\circ} \mathrm{C}$; ${ }^{1} \mathrm{H}$ NMR (300 MHz, DMSO- $\left.d_{6}\right) \delta$ : 9.36 (s, 1H, CH), 8.52 (d, J=7.8 Hz, 1H, ArH), 8.24 (d, $J=8.1 \mathrm{~Hz}, 1 \mathrm{H}, \mathrm{ArH}), 8.07$ (t, $J=7.6 \mathrm{~Hz}, 1 \mathrm{H}, \mathrm{ArH}), 7.93(\mathrm{t}$, $J=7.7 \mathrm{~Hz}, 1 \mathrm{H}, \mathrm{ArH}), 7.71(\mathrm{~d}, J=8.5 \mathrm{~Hz}, 2 \mathrm{H}, \operatorname{ArH}), 7.58$ $(\mathrm{d}, J=8.5 \mathrm{~Hz}, 2 \mathrm{H}, \mathrm{ArH}) ;{ }^{13} \mathrm{C}$ NMR $\left(75 \mathrm{MHz}, \mathrm{DMSO}-d_{6}\right)$ $\delta: 156.58,141.84,140.10,136.99,135.32,135.04,131.81$, $130.23,126.53,126.12,123.42,122.83,122.56$; IR (KBr) $v: 1502.86(\mathrm{C}=\mathrm{N}) \mathrm{cm}^{-1}$; ESI-HRMS calcd for $\mathrm{C}_{15} \mathrm{H}_{10} \mathrm{ClN}_{4} \mathrm{~S}[\mathrm{M}+\mathrm{H}]^{+}$313.0315, found 313.0300.

6-(4-氟代硫苯基)-1,2,4-三氮唑并 $[3,4-a]$ 酞嗪(6c): 合成方法及投料比同上, 产品为白色固体, 产率 $54.5 \%$. m.p. $194 \sim 196{ }^{\circ} \mathrm{C}$; ${ }^{1} \mathrm{H}$ NMR (300 MHz, DMSO-d $\left.d_{6}\right) \delta$ : 9.32 (s, 1H, CH), 8.51 (d, J=7.9 Hz, 1H, ArH), 8.25 (d, $J=8.0 \mathrm{~Hz}, 1 \mathrm{H}, \mathrm{ArH}), 8.08(\mathrm{t}, J=7.6 \mathrm{~Hz}, 1 \mathrm{H}, \mathrm{ArH}), 7.94(\mathrm{t}$, $J=7.7 \mathrm{~Hz}, 1 \mathrm{H}, \mathrm{ArH}), 7.83 \sim 7.73(\mathrm{~m}, 2 \mathrm{H}, \mathrm{ArH}), 7.46 \sim$ 7.34 (m, 2H, ArH); ${ }^{13} \mathrm{C}$ NMR (75 MHz, $\left.\mathrm{CDCl}_{3}\right) \delta: 165.64$, $162.31,157.91,157.89,142.12,139.10,138.11,138.00$, $134.04,130.92,125.38,123.73,122.82,122.52,121.50$, 121.45, 117.10, 116.81; IR ( $\mathrm{KBr}) v: 1502.29(\mathrm{C}=\mathrm{N}) \mathrm{cm}^{-1}$; ESI-HRMS calcd for $\mathrm{C}_{15} \mathrm{H}_{10} \mathrm{FN}_{4} \mathrm{~S}[\mathrm{M}+\mathrm{H}]^{+}$297.0610, found 297.0641 .

\subsubsection{6-氯-四唑并 $[1,5-a]$ 酞嗪(4)的合成}

量取盐酸 $3 \mathrm{~mL}(6 \mathrm{~mol} / \mathrm{L})$, 用 $40 \mathrm{~mL}$ 水稀释, 称取化 合物 $2(2 \mathrm{~g}, 10 \mathrm{mmol})$ 溶解在稀释备用的盐酸水溶液中, 将亚硝酸钠 $(0.7 \mathrm{~g}, 10 \mathrm{mmol})$ 溶解到水 $(15 \mathrm{~mL})$ 中, 缓慢滴 加至事先冰浴的反应体系中, 确保整个反应体系温度在 $5{ }^{\circ} \mathrm{C}$ 下, TLC 跟踪, $3 \mathrm{~h}$ 反应完全, 析出淡棕色沉淀, 抽 滤得滤饼, 乙醇重结晶得纯品 $1.36 \mathrm{~g}$, 化合物为土黄色, 产率 $65 \% \sim 72 \%{ }^{[23]}$. m.p. $188 \sim 190{ }^{\circ} \mathrm{C} ;{ }^{1} \mathrm{H}$ NMR $(300$ $\left.\mathrm{MHz}, \mathrm{CDCl}_{3}\right) \delta: 8.79(\mathrm{~d}, J=7.9 \mathrm{~Hz}, 1 \mathrm{H}), 8.48(\mathrm{~d}, J=8.0$ $\mathrm{Hz}, 1 \mathrm{H}), 8.23 \sim 8.18(\mathrm{~m}, 1 \mathrm{H}), 8.15 \sim 8.09(\mathrm{~m}, 1 \mathrm{H})$.

\section{2 .5 化合物 $7 \mathrm{a} \sim 7 \mathrm{c}$ 和 $8 \mathrm{a} \sim 8 \mathrm{c}$ 的合成通法}

以 $N$-(4-溴苯基)-四唑 $[5,1-a]$ 酞嗪-6-胺(7a)的合成为 例. 将化合物 4 (0.3 g, $1.5 \mathrm{mmol})$ 和对溴苯胺 $(0.31 \mathrm{~g}, 1.8$ $\mathrm{mmol})$ 溶于乙腈 $(25 \mathrm{~mL})$ 中, 再加入碳酸钾 $(0.25 \mathrm{~g}, 1.8$ $\mathrm{mmol})$, 温度升至 $80{ }^{\circ} \mathrm{C}$, 回流 $2 \mathrm{~h}, \mathrm{TLC}\left(V_{\mathrm{DCM}}: V_{\mathrm{MeOH}}=\right.$ $30 ： 1$ 监测, 反应完全后, 减压除去大部分乙腈, 倒入 碎冰水中, 析出沉淀, 抽滤, 水洗至中性, 真空干燥, 用 乙醇进行重结晶得纯品 $7 \mathbf{a}$.

$N$-(4-溴苯基)-四唑 $[5,1-a]$ 酞嗪-6-胺(7a): 淡黄色粉 末, 产率 62.3\%. m.p. 258 260 ${ }^{\circ} \mathrm{C} ;{ }^{1} \mathrm{H}$ NMR (300 MHz, DMSO-d $d_{6} \delta: 9.78(\mathrm{~s}, 1 \mathrm{H}, \mathrm{NH}), 8.75(\mathrm{~d}, J=7.1 \mathrm{~Hz}, 1 \mathrm{H}$,
ArH), $8.63 \sim 8.51(\mathrm{~m}, 1 \mathrm{H}, \mathrm{ArH}), 8.22 \sim 8.02(\mathrm{~m}, 2 \mathrm{H}, \mathrm{ArH})$, $7.81(\mathrm{~d}, J=8.8 \mathrm{~Hz}, 2 \mathrm{H}, \operatorname{ArH}), 7.64$ (d, $J=8.8 \mathrm{~Hz}, 2 \mathrm{H}$, ArH); ${ }^{13} \mathrm{C}$ NMR (75 MHz, DMSO- $\left.d_{6}\right) \delta: 151.52,140.53$, $138.77,134.43,132.70,132.03,125.46,124.71,124.55$, $122.38,120.55,116.28$; IR $(\mathrm{KBr}) v$ : $3282.66(\mathrm{~N}-\mathrm{H})$, $1505.71(\mathrm{C}=\mathrm{N}) \mathrm{cm}^{-1}$; ESI-HRMS calcd for $\mathrm{C}_{14} \mathrm{H}_{10} \mathrm{BrN}_{6}$ $[\mathrm{M}+\mathrm{H}]^{+}$341.0150, found 341.0139.

$N$-(4-氯苯基)-四唑 $[5,1-a]$ 酞嗪-6-胺(7b): 制备方法 及投料比同上，产品为黄色粉末，产率 $58.1 \%$. m.p. $258 \sim 262{ }^{\circ} \mathrm{C} ;{ }^{1} \mathrm{H}$ NMR (300 MHz, DMSO- $\left.d_{6}\right) \delta: 9.78(\mathrm{~s}$, $1 \mathrm{H}, \mathrm{NH}), 8.81 \sim 8.70(\mathrm{~m}, 1 \mathrm{H}, \mathrm{ArH}), 8.62 \sim 8.50(\mathrm{~m}, 1 \mathrm{H}$, ArH), 8.19 8.02 (m, 2H, ArH), $7.86(\mathrm{~d}, J=8.9 \mathrm{~Hz}, 2 \mathrm{H}$, ArH), 7.49 (d, $J=8.9 \mathrm{~Hz}, 2 \mathrm{H}, \mathrm{ArH}) ;{ }^{13} \mathrm{C} \mathrm{NMR}(75 \mathrm{MHz}$, DMSO- $\left.d_{6}\right) \delta: 151.45,140.41,138.25,134.29,132.57$, 129.01, 128.21, 125.39, 124.61, 124.11, 122.29, 120.41; IR $(\mathrm{KBr}) \quad v: 3336.28(\mathrm{~N}-\mathrm{H}), 1514.29(\mathrm{C}=\mathrm{N}) \mathrm{cm}^{-1}$; ESI-HRMS calcd for $\mathrm{C}_{14} \mathrm{H}_{10} \mathrm{ClN}_{6}[\mathrm{M}+\mathrm{H}]^{+}$297.0655, found 297.0641 .

$N$-(4-氟苯基)-四唑 [5,1- $a$ ]酞嗪-6-胺(7c)：制备方法 及投料比同上，产品为淡黄色固体，产率 $46.3 \%$. m.p. 272 $274{ }^{\circ} \mathrm{C} ;{ }^{1} \mathrm{H}$ NMR (300 MHz, DMSO- $\left.d_{6}\right) \delta: 9.72(\mathrm{~s}$, $1 \mathrm{H}, \mathrm{NH}), 8.71$ (d, $J=7.1 \mathrm{~Hz}, 1 \mathrm{H}, \mathrm{ArH}), 8.53$ (d, $J=7.6$ $\mathrm{Hz}, 1 \mathrm{H}, \mathrm{ArH}), 8.08$ (t, $J=8.5 \mathrm{~Hz}, 2 \mathrm{H}, \mathrm{ArH}), 7.80$ (dd, $J=$ 8.6, $4.9 \mathrm{~Hz}, 2 \mathrm{H}, \mathrm{ArH}), 7.30$ (t, $J=8.7 \mathrm{~Hz}, 2 \mathrm{H}, \mathrm{ArH}) ;{ }^{13} \mathrm{C}$ NMR (75 MHz, DMSO- $\left.d_{6}\right) \delta: 160.81,158.60,157.62$, $151.88,140.42$, 135.52, 134.32, 132.65, 125.33, 125.16, $125.06,124.65,122.29,120.43,116.02,115.73$; IR (KBr) v: $3274.56(\mathrm{~N}-\mathrm{H}), 1511.43(\mathrm{C}=\mathrm{N}) \mathrm{cm}^{-1}$; ESI-HRMS calcd for $\mathrm{C}_{14} \mathrm{H}_{10} \mathrm{FN}_{6}[\mathrm{M}+\mathrm{H}]^{+}$281.0951, found 281.0939.

6-(4-溴代硫苯基)-四唑 $[5,1-a]$ 酞嗪 $(\mathbf{8 a})$ : 制备方法 及投料比同上，产品为白色粉末，产率 $71.1 \%$. m.p. $240 \sim 242{ }^{\circ} \mathrm{C} ;{ }^{1} \mathrm{H}$ NMR (300 MHz, DMSO- $\left.d_{6}\right) \delta: 8.66(\mathrm{~s}$, $1 \mathrm{H}, \mathrm{ArH}), 8.44$ (d, $J=7.3 \mathrm{~Hz}, 1 \mathrm{H}, \operatorname{ArH}), 8.21$ (d, $J=7.6$ $\mathrm{Hz}, 1 \mathrm{H}, \mathrm{ArH}), 8.15$ (d, $J=6.9 \mathrm{~Hz}, 1 \mathrm{H}, \mathrm{ArH}), 7.79$ (d, $J=$ $7.6 \mathrm{~Hz}, 2 \mathrm{H}, \mathrm{ArH}), 7.72$ (s, 2H, ArH); ${ }^{13} \mathrm{C}$ NMR (75 MHz, DMSO- $\left.d_{6}\right) \delta: 159.89,141.98,137.82,135.68,133.61$, $133.35,126.17,125.89,124.92,124.72,124.44,121.42$; IR $(\mathrm{KBr}) v: 1474.29(\mathrm{C}=\mathrm{N}) \mathrm{cm}^{-1}$; ESI-HRMS calcd for $\mathrm{C}_{14} \mathrm{H}_{9} \mathrm{BrN}_{5} \mathrm{~S}[\mathrm{M}+\mathrm{H}]^{+}$359.9762, found 359.9729.

6-(4-氯代硫苯基)-四唑 $[5,1-a]$ 酞嗪 $(8 b)$ : 制备方法 及投料比同上，产品为白色固体，产率 $63.1 \%$. m.p. $232 \sim 236{ }^{\circ} \mathrm{C} ;{ }^{1} \mathrm{H}$ NMR (300 MHz, DMSO- $\left.d_{6}\right) \delta: 8.66(\mathrm{~d}$, $J=7.4 \mathrm{~Hz}, 1 \mathrm{H}, \mathrm{ArH}), 8.45$ (d, $J=7.7 \mathrm{~Hz}, 1 \mathrm{H}, \mathrm{ArH}), 8.22$ (d, $J=7.5 \mathrm{~Hz}, 1 \mathrm{H}, \mathrm{ArH}), 8.15(\mathrm{~d}, J=7.5 \mathrm{~Hz}, 1 \mathrm{H}, \mathrm{ArH})$, 7.79 (d, $J=7.8 \mathrm{~Hz}, 2 \mathrm{H}, \operatorname{ArH}), 7.67$ (d, $J=7.6 \mathrm{~Hz}, 2 \mathrm{H}$, 
ArH); ${ }^{13} \mathrm{C}$ NMR (75 MHz, DMSO- $\left.d_{6}\right) \delta: 159.99,141.96$, 137.67, 135.98, 135.67, 133.60, 130.43, 126.13, 125.31, 124.90, 124.40, 121.39; IR (KBr) v: $1480(\mathrm{C}=\mathrm{N}) \mathrm{cm}^{-1}$; ESI-HRMS calcd for $\mathrm{C}_{14} \mathrm{H}_{9} \mathrm{ClN}_{5} \mathrm{~S}[\mathrm{M}+\mathrm{H}]^{+}$314.0267, found 314.0258 .

6-(4-氟代硫苯基)-四唑 $[5,1-a]$ 酞嗪(8c): 制备方法 及投料比同上, 产品为白色固体, 产率 $63.1 \%$. m.p. $232 \sim 236{ }^{\circ} \mathrm{C} ;{ }^{1} \mathrm{H}$ NMR $\left(300 \mathrm{MHz}, \mathrm{DMSO}-d_{6}\right) \delta: 8.64(\mathrm{~d}$, $J=7.7 \mathrm{~Hz}, 1 \mathrm{H}, \mathrm{ArH}), 8.44$ (d, $J=7.9 \mathrm{~Hz}, 1 \mathrm{H}, \mathrm{ArH}), 8.22$ (t, $J=7.2 \mathrm{~Hz}, 1 \mathrm{H}, \mathrm{ArH}), 8.13$ (t, $J=7.6 \mathrm{~Hz}, 1 \mathrm{H}, \mathrm{ArH}), 7.82$ (dd, $J=8.3,5.4 \mathrm{~Hz}, 2 \mathrm{H}, \operatorname{ArH}), 7.46(\mathrm{t}, J=8.7 \mathrm{~Hz}, 2 \mathrm{H}$, ArH); ${ }^{13} \mathrm{C}$ NMR (75 MHz, DMSO- $d_{6}$ ) $\delta: 165.57,162.27$, $160.39,141.90,138.72,138.61,135.62,133.57,126.04$, $124.88,124.31,121.72,121.68,121.30,117.78,117.48$; IR $(\mathrm{KBr}) v: 1494.29(\mathrm{C}=\mathrm{N}) \mathrm{cm}^{-1}$; ESI-HRMS calcd for $\mathrm{C}_{14} \mathrm{H}_{9} \mathrm{FN}_{5} \mathrm{~S}[\mathrm{M}+\mathrm{H}]^{+}$298.0563, found 298.0549.

\section{2 抗惊厥药理实验}

药理实验方法参照美国卫生研究院抗癫㾁药物开 发计划的抗癫㾁药物笁选程序 ${ }^{[24,25]}$. 采用最大电惊厥 (MES) 实验评价化合物抗惊厥药理活性, 此方法能够迅 速、全面地篮选化合物的抗惊厥活性. 实验采用昆明种 小鼠, 体重 18 22 g (鼠龄周 4 5 周)为测试动物, 雌雄 各半. 实验前一周饲养于 $20 \sim 25{ }^{\circ} \mathrm{C}$ 、相对湿度 $45 \%$ $65 \%$ 的实验室中, 可以自由采食和饮水. 抗惊厥药理实 验主要分成两个阶段, I 期和 II 期药理实验; 在最大电惊 厥实验中, 使用 $50 \mathrm{~mA}, 60 \mathrm{~Hz}$ 的交流电, 小鼠耳电极通 电 $0.2 \mathrm{~s}$ 出现后肢强直的视为惊厥, 正式实验前一天预 笁小鼠，出现惊厥的小鼠用于正式实验.

\subsection{1 小鼠体内 $\mathrm{I}$ 期实验}

在 I 期药理实验中, 将小鼠随机分组, 每组 3 只. 将 药物溶解在适量二甲基亚砜(DMSO)中, 分别以 30, 100, $300 \mathrm{mg} \cdot \mathrm{kg}^{-1}$ 不同的剂量, 给与小白鼠腹腔注射; 给药 $0.5 \mathrm{~h}$ 后进行最大电惊厥实验, 记录不同剂量下的抗惊 厥小鼠的个数, 以此来初步评价化合物的抗惊厥活性.

\subsection{2 小鼠体内 II 期实验}

为定量评价 I 期药理实验中耖选出的活性化合物, 在 II 期药理实验中主要测定其抗惊厥的半数有效量, 并 计算其 95\%可信限. 阳性对照药依然选用临床上常用抗 癫痫药卡马西平。

辅助材料(Supporting Information) 化合物 $\mathbf{5 a} \sim \mathbf{5 g}$, $6 \mathrm{a} \sim 6 \mathrm{c}, 7 \mathrm{a} \sim 7 \mathrm{c}, 8 \mathrm{a} \sim 8 \mathrm{c}$ 的核磁共振氢谱、碳谱和高分辨
质谱图谱. 这些材料可以免费从本刊网站(http://siocjournal.cn/)上下载.

\section{References}

[1] Gasior, M.; Carter, R. B.; Goldberg, S. R.; Witkin, J. M.; Pharmacol, J. Exp. Ther. 1997, 282, 544.

[2] Mccormick, D. A.; Contreras, D. Annu. Rev. Physiol. 2001, 63, 815.

[3] Strine, T. W.; Kobau, R.; Chapman, D. P.; Thurman, D. J.; Price, P.; Balluz, L. S. Epilepsia 2005, 46, 1133.

[4] Sirven, J. I.; Noe, K.; Hoerth, M.; Drazkowski, J. Mayo. Clin. Proc. 2012, 87, 879 .

[5] Kwan, P.; Brodie, M. J. N. Engl. J. Med. 2000, 342, 315.

[6] Penovich, P. E.; Willmore, L. J. Epilepsia 2009, 50, 38.

[7] Bialer, M.; Johannessen, S. I.; Kupferberg, H. J.; Levy, R. H.; Perucca, E.; Tomson, T. Epilepsy Res. 2007, 73, 2.

[8] Bootsma, H. P.; Ricker, L.; Hekster, Y. A.; Hulsman, J.; Lambrechts, D.; Majoie, M.; Schellekens, A.; Krom, D. M.; Aldenkamp, A. P. Seizure 2009, 18, 328.

[9] Ramaiya, S.; Sundararaj, K. G.; Somasundaram, R.; Joseph, T. L. Eur. J. Med. Chem. 2002, 37, 793.

[10] Guo, L. J.; Wei, C. X.; Jia, J. H.; Zhao, L. M.; Quan, Z. S. Eur. J. Med. Chem. 2009, 44, 954.

[11] Sun, X. Y.; Wei, C. X.; Deng, X. Q.; Sun, Z. G.; Quan, Z. S. Pharmacol. Rep. 2010, 62, 273.

[12] Wang, S. B.; Deng, X. Q.; Zheng, Y.; Yuan, Y. P.; Quan, Z. S.; Guan, L. P. Eur. J. Med. Chem. 2012, 56, 139

[13] Zhu, Z. S.; Wang, S. B.; Deng, X. Q.; Liu, D. C.; Quan, Z. S. Lett. Drug Des. Dis. 2014, 11, 628.

[14] Wang, S. B.; Deng, X. Q.; Liu, D. C.; Zhang, H. J.; Quan, Z. S. Med. Chem. Res. 2014, 23, 4619.

[15] Tan, Y. D.; He, X. Y.; Rao, B. Q.; Cheng, B. B.; Song, M. X.; Deng, X. Q. Chin. J. Org. Chem. 2016, 36, 2449 (in Chinese). (谭月德, 何晓艳, 饶宝奇, 程涁彬, 宋明霞, 邓先清, 有机化学, 2016, 36, 2449.)

[16] Zhang, L.; Guan, L. P.; Sun, X. Y.; Wei, C. X.; Chai, K. Y.; Quan, Z. S. Chem. Biol. Drug Des. 2009, 73, 313.

[17] Deng, X. Q.; Wei, C. X.; Song, M. X.; Quan, Z. S. Arzneim. Forsch. 2010, 60, 587.

[18] Bian, M.; Deng, X. Q.; Gong, G. H.; Wei, C. X.; Quan, Z. S. J. Enzyme Inhib. Med. Chem. 2013, 28, 792.

[19] Street, L. J.; Sternfeld, F.; Jelley, R. A.; Reeve, A. J.; Carling, R. W.; Moore, K. W.; McKernan, R. M.; Sohal, B.; Cook, S.; Pike, A.; Dawson, G. R.; Bromidge, F. A.; Wafford, K. A.; Seabrook, G. R.; Thompson, S. A.; Marshall, G.; Pillai, G. V.; Castro, J. L.; Atack, J. R.; MacLeod, A. M. J. Med. Chem. 2004, 47, 3642.

[20] Wu, Y.; Sun, L. P.; Ma, L. X.; Che, J.; Song, M. X. Chem. Biol. Drug Des. 2013, 81, 591.

[21] Wang, C. J.; Cao, Q. P.; Yang, H.; Song, P. P.; Xue, D. Q.; Cui, F.; Gu, Y. F.; Zhang, X. S.; Tian, Y. N.; Zhang, Q. R.; Liu, H. M. Chin. J. Org. Chem. 2016, 36, 1627 (in Chinese). (王超杰，曹钦坡，杨慧，宋攀攀，薛登启，崔飞，顾一飞，张孝 松，田亚楠，张秋荣，刘宏民，有机化学, 2016, 36, 1627.)

[22] Xue, D. Q.; Zhang, X. Y.; Wang, C. J.; Ma, L. Y.; Zhu, N.; He, P.; Shao, K. P.; Chen, P. J.; Gu, Y, F.; Zhang, X. S.; Wang, C. F.; Ji, C. H.; Zhang, Q. R.; Liu, H. M. Eur. J. Med. Chem. 2014, 85, 235.

[23] Bian, M.; Deng, X. Q.; Gong, G. H.; Wei, C. X.; Quan, Z. S. J. Enzyme Inhib. Med. Chem. 2013, 28, 792.

[24] Okada, R.; Negishi, N.; Nagaya, H. Brain Res. 1989, 480, 384.

[25] Liu, D. C.; Deng, X. Q.; Wang, S. B.; Quan, Z. S. Arch. Pharm. (Weinheim, Ger.) 2014, 347, 269. 